\title{
SYNTHESIS OF SOME NEW 3-ARYL-1-( 4,6-DIMETHYL-2-PYRIMIDINYL)-4- FORMYLPYRAZOLES USING VILSMEIER HAACK REACTION
}

\author{
Om Prakash*, Ravi Kumar, Vikas Bhardwaj and Pawan K. Sharma \\ *Department of Chemistry, Kurukshetra University, Kurukshetra, Haryana-1361 19 (India ). \\ e-mail: search(a)vidva.kuk.ernet.in and chem.@granth.kuk.ernet.in
}

\begin{abstract}
:
Synthesis of some new 3-aryl-1-(4,6-dimethyl-2-pyrimidinyl)-4-formylpyrazoles has been accomplished by the reaction of 4,6-dimethyl-2-pyrimidinylhydrazones of various acetophenones with Vilsmeier - Haack reagent $\left(\mathrm{POCl}_{3} / \mathrm{DMF}\right)$.
\end{abstract}

\section{Introduction :}

The use of Vilsmeier - Haack reagent ( $\left.\mathrm{POCl}_{3} / \mathrm{DMF}\right)$ to bring about formylation and related transformations from aromatic and conjugated cyclic compounds is well established.' Besides this, the reagent is also employed for effecting various chemical transformations from other classes of compounds. ${ }^{2}$ A notable example that finds an interesting and significant application in heterocyclic chemistry is the synthesis of pyrazoles from the reaction of hydrazones and semicarbazones with Vilsmeier reagent (Eq. 1$)^{3}$

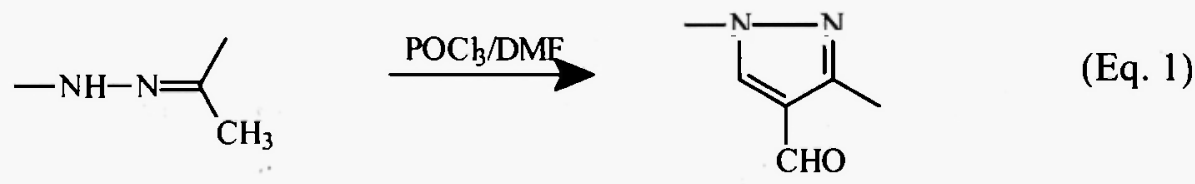

The wide range of biological activities such as antibacterial ${ }^{4}$, antifungal ${ }^{5}$ etc. associated with pyrazoles ${ }^{6}$ and pyrimidines ${ }^{7}$, coupled with the fact that much attention has not been paid to study the scope of this important modification of Vilsmeier reaction for the synthesis of pyrazole derivatives containing heterocyclic moieties ${ }^{8}$ such as pyrimidines etc., prompted us to undertake the synthesis of some new pyrimidinylpyrazoles (3a-3f) by using $\mathrm{POCl}_{3} / \mathrm{DMF}$. 


\section{Results and Discussion :}

To check the feasibility of this approach for the synthesis of pyrimidinylpyrazoles, 4,6-dimethyl-2pyrimidinylhydrazone (2a) of acetophenone was stirred with 3 equivalents of phosphorous oxychloride in DMF at a temperature of $50^{\circ}-60^{\circ} \mathrm{C}$ for 2 hours. The reaction afforded the desired product 3-phenyl-1-( 4,6-dimethyl-2-pyrimidinyl)-4-formylpyrazole (3a) in $65 \%$ yield.

The method was found to be general as hydrazones $\mathbf{2 b}-\mathbf{2} \mathbf{f}$ of variously substituted acetophenones were converted to the corresponding pyrimidinylpyrazoles (3b-3f) in yields ranging from $65 \%-85 \%$ ( Scheme I) The structures of the products were confirmed by their IR, 'HNMR and HRMS data. The results of the reaction along with the chacterisation data are summazied in table 1 .<smiles>CCCCCCCCCCCCCCCCCCCOC(C)=O</smiles>

1<smiles>[R]c1ccc(-c2nn(-c3nc(C)cc(C)n3)cc2C=O)cc1</smiles>

3<smiles>[R]c1ccc(C(C)=N)cc1</smiles>

2

$2,3 \quad \mathrm{R}$

a $\mathrm{H}$

b $\quad \mathrm{NO}_{2}$

c $\mathrm{Cl}$

d $F$

e $\mathrm{OH}$

f $\mathrm{Me}$

\section{$\underset{\mathrm{DMF} / \mathrm{POCl}_{3}}{\longrightarrow}$}

Scheme-1

The new pyrimidinylpyrazoles (3a-3f) obtained from this study are important precursors for fused bispyrazoles. The synthesis of the latter involving hypervalent iodine reagents are under progress and will be published in detail in our future communications.

\section{Experimental:}

Melting points were recorded in open capillaries and are uncorrected. The ${ }^{1} \mathrm{H}$ NMR spectra of the products were recorded on Bruker spectrophotometer at $300 \mathrm{MHz}$.

The requisite 4,6-dimethyl-2-pyrimidinylhydrazones (2a-2f) were prepared by the standard procedure invoving condensation of 4,6-dimethyl-2-hydrazinopyrimidine (1) with corresponding acetophenones in ethanol. ${ }^{9} \quad 4,6$-Dimethyl-2-hydrazinopyrimidine (1) was synthesized according to the literature procedure commencing with urea and acetylacetone. ${ }^{10}$ 
Preparation of 3-aryl-l-( 4,6-dimethyl-2-pyrimidinyl)-4-formylpyrazoles(3a-3f) : -

General Procedure:- To the Vilsmeier-Haack reagent prepared by dissolving $\mathrm{POCl}_{3}(3 \mathrm{mmol}, 0.28 \mathrm{ml})$ in 20-30 $\mathrm{ml} \mathrm{DMF} \mathrm{appropriate} \mathrm{hydrazone} 2(1 \mathrm{mmol})$ was added and the reaction mixture was stirred at $50^{\circ}-60^{\circ}$ $\mathrm{C}$ for a period of 2-3 hours. The reaction mixture was then poured over ice cold water. The solid that separated on neutralization with $\mathrm{NaHCO}_{3}$ was filtered, washed with water and recrystallised from $\mathrm{EtOH}$ to afford pure 3-aryl-1-( 4,6-dimethyl-2-pyrimidinyl)-4-formylpyrazole 3 (Table 1 ).

\section{Antibacterial testing:}

All the compounds (3a-3f) were tested against two organisms: Staphylococcus aureus (gram - positive) and Pseudomonas aeruginosa (gram - negative) at concentration of $5 \%$ in chloroform by Filter Paper Disk Method." Both of the organisms were cultured in nutrient agar medium. However, none of the compounds showed any significant activity.

Table 1: Spectral and Physical data of 3<smiles>[R]c1ccc(-c2nn([Al])cc2C=O)cc1</smiles>

3

\begin{tabular}{|c|c|c|c|c|c|c|}
\hline $\begin{array}{l}\text { Sr. } \\
\text { No. }\end{array}$ & Compd. & $\begin{array}{l}\text { M.Pt. } \\
\left({ }^{\circ} \mathrm{C}\right)\end{array}$ & $\begin{array}{l}\text { Yield } \\
(\%)\end{array}$ & $\begin{array}{c}\mathrm{IR}(\mathrm{KBr}) \\
\max . \\
\mathrm{cm}^{-1}\end{array}$ & $\begin{array}{c}\text { 'H NMR }\left(\mathrm{CDCl}_{3}\right) \\
(\mathrm{ppm})\end{array}$ & $\begin{array}{l}\text { HRMS }(\mathrm{m} / \mathrm{z}) \\
\left(\mathrm{M}^{+}, \%\right)\end{array}$ \\
\hline 1. & $3 \mathbf{a}$ & $120-121$ & 65 & 1694 & $\begin{array}{l}2.59\left(\mathrm{~s}, 6 \mathrm{H}, \mathrm{C}_{4}-\mathrm{CH}_{3}, \mathrm{C}_{6}-\mathrm{CH}_{3}\right), 7.04 \\
\left(\mathrm{~s}, 1 \mathrm{H}, \mathrm{C}_{5}-\mathrm{H}\right), 7.46-7.50(\mathrm{~m}, 3 \mathrm{H}), 7.65- \\
7.68(\mathrm{~m}, 2 \mathrm{H}), 9.26\left(\mathrm{~s}, 1 \mathrm{H}, \mathrm{C}_{5} \quad-\mathrm{H}\right), 10.07 \\
(\mathrm{~s}, 1 \mathrm{H}, \mathrm{CHO})\end{array}$ & $\begin{array}{l}278.1165 \\
(100)\end{array}$ \\
\hline 2. & $3 \mathbf{b}$ & $145-146$ & 83 & 1692 & $\begin{array}{l}2.62\left(\mathrm{~s}, 6 \mathrm{H}, \mathrm{CH}_{4} \mathrm{CH} 3\right), 7.09\left(\mathrm{~s}, 1 \mathrm{H}, \mathrm{C}_{5^{-}}\right. \\
\mathrm{H}), 6.19-6.22\left(\mathrm{~d}, 2 \mathrm{H}, \mathrm{C}_{2} \quad-\mathrm{H}, \mathrm{C}_{6} \quad \mathrm{H}\right), 6.32- \\
6.35\left(\mathrm{~d}, 2 \mathrm{H}, \mathrm{C}_{3}-\mathrm{H}, \mathrm{C}_{5}-\mathrm{H}\right), 9.32 \\
\left(\mathrm{~s}, 1 \mathrm{H}, \mathrm{C}_{5}-\mathrm{H}\right), 10.1(\mathrm{~s}, 1 \mathrm{H},-\mathrm{CHO})\end{array}$ & $323.1017(80)$ \\
\hline 3. & $3 c$ & $150-151$ & 80 & 1692 & $\begin{array}{l}2.60\left(\mathrm{~s}, 6 \mathrm{H}, \mathrm{C}_{4}-\mathrm{CH}_{3}\right), 7.05\left(\mathrm{~s}, 1 \mathrm{H}, \mathrm{C}_{5}-\mathrm{H}\right) \\
7.44-7.47\left(\mathrm{~d}, 2 \mathrm{H}, \mathrm{C}_{2} \quad \mathrm{H}, \mathrm{C}_{6}-\mathrm{H}\right), 7.87-7.90 \\
\left(\mathrm{~d}, 2 \mathrm{H}, \mathrm{C}_{3} \quad-\mathrm{H}, \mathrm{C}_{5}-\mathrm{H}\right), 9.27\left(\mathrm{~s}, 1 \mathrm{H}, \mathrm{C}_{5}-\right. \\
\mathrm{H}), 10.05(\mathrm{~s}, 1 \mathrm{H},-\mathrm{CHO})\end{array}$ & $\begin{array}{c}312.0770 \\
(100)\end{array}$ \\
\hline 4. & 3d & $100-101$ & 77 & 1691 & $\begin{array}{l}2.60\left(\mathrm{~s}, 6 \mathrm{H}, \mathrm{C}_{4}-\mathrm{CH}_{3}\right), 7.06\left(\mathrm{~s}, 1 \mathrm{H}, \mathrm{C}_{5}-\mathrm{H}\right), \\
7.17-7.20\left(\mathrm{~d}, 2 \mathrm{H}, \mathrm{C}_{2}-\mathrm{H}, \mathrm{C}_{6}-\mathrm{H}\right), 7.91-7.94 \\
\left(\mathrm{~d}, 2 \mathrm{H}, \mathrm{C}_{3}-\mathrm{H}, \mathrm{C}_{5}-\mathrm{H}\right), 9.20\left(\mathrm{~s}, 1 \mathrm{H}, \mathrm{C}_{5}-\mathrm{H}\right), \\
10.05(\mathrm{~s}, \mathrm{lH},-\mathrm{CHO})\end{array}$ & $\begin{array}{c}296.1077 \\
(100)\end{array}$ \\
\hline 5. & $3 \mathrm{e}$ & $248-249$ & 71 & 1699 & $\begin{array}{l}2.59\left(\mathrm{~s}, 6 \mathrm{H}, \mathrm{C}_{4}-\mathrm{CH}_{4}\right), 7.03\left(\mathrm{~s}, 1 \mathrm{H}, \mathrm{C}_{5}-\mathrm{H}\right) \\
7.70-7.93\left(\mathrm{~d}, 2 \mathrm{H}, \mathrm{C}_{2}-\mathrm{H}, \mathrm{C}_{6}-\mathrm{H}\right), 6.90-6.93 \\
\left(\mathrm{~d}, 2 \mathrm{H}, \mathrm{C}_{3} \quad-\mathrm{H}, \mathrm{C}_{5}-\mathrm{H}\right), 9.24\left(\mathrm{~s}, 1 \mathrm{H}, \mathrm{C}_{5}-\right. \\
\mathrm{H}), 10.04(\mathrm{~s}, 1 \mathrm{H},-\mathrm{CHO})\end{array}$ & $\begin{array}{c}294.1110 \\
(100)\end{array}$ \\
\hline 6. & $3 \mathrm{f}$ & $151-152$ & 68 & 1679 & $\begin{array}{l}2.42\left(\mathrm{~s}, 3 \mathrm{H}, \mathrm{C}_{4}-\mathrm{CH}_{3}\right), \quad 2.59\left(\mathrm{~s}, 6 \mathrm{H}, \mathrm{C}_{4^{-}}\right. \\
\left.\mathrm{CH}_{3}\right), 7.04\left(\mathrm{~s}, \mathrm{IH}, \mathrm{C}_{5}-\mathrm{H}\right), 7.75-7.77 \\
\left(\mathrm{~d}, 2 \mathrm{H}, \mathrm{C}_{2} \quad-\mathrm{H}, \mathrm{C}_{6}-\mathrm{H}\right), 7.28-7.30 \\
\left(\mathrm{~d}, 2 \mathrm{H}, \mathrm{C}_{3}-\mathrm{H}, \mathrm{C}_{5}-\mathrm{H}\right), 9.27\left(\mathrm{~s}, \mathrm{IH}, \mathrm{C}_{5}-\right. \\
\mathrm{H}), 10.06(\mathrm{~s}, 1 \mathrm{H},-\mathrm{CHO})\end{array}$ & $292.1569(9.7)$ \\
\hline
\end{tabular}




\section{Acknowledgement:}

We are thankful to CSIR and DRDO for providing the financial assistance to accomplish this work. We are also grateful to Mass Spectrometry Facility, University of Califormia, San Francisco which is supported by Biomedical Research Technology Programme for providing mass spectra and to Mr. Avtar Singh, Department of Biotechnology, Kurukshetra University, Kurukshetra for performing antibacterial testing.

\section{References:}

1. (a) G. Jones, Organic Reactions, Vol. 49, Published by John Wiley \& Sons, Inc. and references therein. (b) A. Nohara, T. Umetani and Y. Sanno, Tetrahedron, 30, 3553 (1974).

2. (a) G. Jones, Organic Reactions, Vol. 56, Published by John Wiley \& Sons, Inc. and references therein (b) M .A. Kira, Afdel-Raeman and K Z Gadalla, Tetrahedron Letters, 2, 109 ( 1969).

3. (a) M. Bernard, E. Hulley, H. Molenda, K. Stochla, U. Wrzeciono, Pharmazie, 41, 560,1986. (b) M.A.Kira, Aboul-Enein, M.I.Korkov, J.Heterocycl. Chem.,7,25,1970.

4. P.Calabresi and R.E.Parks; in ,The Pharmacological Basis of Therapeutics", ed. L.S.Goodman and A.Gilman , Macmillan ,NewYork , $5^{\text {th }}$ edn. , 1975, 102.

5. N.A.Devi, K.Khuman, R.K.T.Singh and L.W.Singh , Indian J Het Chem. ,7 , 1998, 193.

6. L. S. Goodman and A . Gilman "The Pharmacological Basis of Therapeutics", Macmillan, NewYork (1980).

7. (a) K. L. Wierzchowsk, E. Litonskao, and D. Shuger, J.Am.Chem. Soc., 87,4621 (1965),(b)

G.Schmidt, Ann. Rev., Biochem.,19, 149 (1950)

8. P. K. Sharma, K. Singh, S. N. Dhawan and S. P. Singh, Indian J Chem.,41A , 2071-2075 (2002).

9. (a) H. Vanderhaeghe and Mr. Claesen, (Univ. Louvain) Bull. Soc. Chim Belg. 68, 30-46 (1959).

(b) Luciano Fabbrini (Univ. Floreuce), Gujj Chim. Ital.87,1293-302 (1957).

10. (a) G. M. Kosloapoff and C. H.Roy, J. Org. Chem., 26,1895 (1961), (b) Mastukawa and Ohta, J. Pham. Soc. , Japan, 69, 491, (1949).

11. Microbiological Applications: A Laboratory Manual of General Microbiology (Fifth Edition), Harold J. Benson, W.C.Brown Publishers, 1990.

\section{Received on April 15, 2003.}

